\title{
Prevalencia de diabetes tipo 2 y obesidad en dos poblaciones aborígenes de Chile en ambiente urbano
}

\author{
Elena Carrasco $\mathbf{P}^{1 a}$, Francisco Pérez $\mathbf{B}^{2 b}$, Bárbara Angel $\mathbf{B}^{2 c}$, \\ Cecilia Albala $\mathrm{B}^{2}$, J Luis Santos $\mathrm{M}^{2 \mathrm{~b}}$, Gladys Larenas $\mathrm{Y}^{3}$, \\ Domingo Montalvo $\mathrm{V}^{4}$. \\ Prevalence of type 2 diabetes and obesity \\ in two Chilean aboriginal populations \\ living in urban zones
}

Background: The prevalence of cardiovascular risk factors is increasing in aboriginal populations in Chile. Aim: To study the prevalence of obesity, type 2 diabetes and serum lipids in two aboriginal populations, Mapuche and Aymara, that were transferred from a rural to a urban environment. Subjects and Methods: Two groups of subjects over 20 years were analyzed, Mapuche and Aymara. The Mapuche group was formed by 42 men and 105 women, living in four urban communities of Santiago, and an Aymara group formed by 42 men and 118 women, living in Arica, in Northern Chile. Anthropometric measurements, blood pressure, lipid profile, oral glucose tolerance test, fasting insulin and serum leptin were determined. Results: The prevalence of type 2 diabetes was 6.9\% in Aymara and 8.2\% in Mapuche subjects. The frequency of glucose intolerance was similar in both groups, but greater among men. A total blood cholesterol over $200 \mathrm{mg} / \mathrm{dl}$ was observed in 43.1\% of Aymara and 27.9\% of Mapuche subjects $(\mathrm{p}<0.008)$. Serum triglycerides over $150 \mathrm{mg} / \mathrm{dl}$ were observed in 16.9 and $23.1 \%$ of Aymara and Mapuche individuals, respectively $(\mathrm{p}=\mathrm{NS})$. Conclusions: The prevalence of type 2 diabetes and dyslipidemia in urban aboriginal populations is higher than that of their rural counterparts. A possible explanation for these results are changes in lifestyles that come along with urbanization, characterized by a high consumption of saturated fat and refined sugars and a low level of physical activity (Rev Méd Chile 2004; 132: 1189-97).

(Key Words: Diabetes Mellitus, type II; Hypercholesterolemia; Hyperlipidemia; Indians, South American)

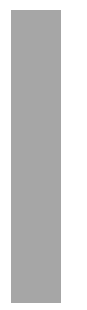

\footnotetext{
Recibido el 15 de marzo, 2004. Aceptado en versión corregida el 19 de agosto, 2004.

${ }^{1}$ Unidad de Diabetes, Hospital San Juan de Dios. Departamento de Medicina. Facultad de Medicina Occidente, Universidad de Chile. ${ }^{2}$ Laboratorio de Epidemiología Genética, Instituto de Nutrición y Tecnología de los Alimentos (INTA), Universidad de Chile. ${ }^{3}$ Departamento de Endocrinología, Universidad de La Frontera, Hospital Regional de Temuco. ${ }^{4}$ Departamento de Salud, Universidad de Tarapacá.

aMSc Nutrición

${ }^{b}$ Doctor en Ciencias

'MSc Biología de la Reproducción
}

Correspondencia a: Elena Carrasco P. Real Audiencia 1361,

San Miguel, Santiago, Chile. Zip Code: 7140596. Fax: 56-2-

68254 65. E mail: ecarrasc@med.uchile.cl 
L as enfermedades crónicas asociadas a la nutrición (ECN) son en la actualidad las principales causas de enfermedad y muerte en la mayor parte del mundo ${ }^{1}$. En Chile, las enfermedades cardiovasculares y el cáncer representan las mayores amenazas para la salud de la población, ambas relacionadas en forma directa o indirecta a los hábitos nutricionales, en asociación al perfil genético característico de las personas ${ }^{2-6}$.

La obesidad es una enfermedad crónica tratable y prevenible, caracterizada por la acumulación de un exceso de grasa en el cuerpo, que provoca efectos adversos severos ${ }^{1}$. Al grave daño sobre la salud que produce por sí misma, se suma la asociación con patologías graves como la diabetes tipo 2, la hipertensión, las complicaciones cardiovasculares y algunos tipos de cáncer ${ }^{1,2}$. Desde el punto de vista de la salud pública, en la actualidad la obesidad constituye la patología nutricional más importante en el país, con una alta prevalencia y en acelerado aumento en todos los grupos etáreos, especialmente en mujeres de bajos recursos ${ }^{4}$.

La diabetes tipo 2 es una de las comorbilidades más asociadas al exceso de grasa corporal. En un reciente informe de la $\mathrm{OMS}^{8}$ se destacan diversas cifras de prevalencia de diabetes tipo 2 en América Latina y el Caribe. La tasa más elevada correspondió a Barbados (16,4\%), seguida por Cuba con $14,8 \%$, en tanto que la más baja fue registrada en 1998 entre la población Aymara de una zona rural de Chile $(1,5 \%)^{9}$. En la mayoría de los países la prevalencia de diabetes es más elevada en mujeres que en hombres.

De acuerdo a las estimaciones de la Organización Mundial de la Salud para las Américas para el año $2000^{8}$, Chile se encuentra en el grupo de países con las mayores prevalencias de diabetes en poblaciones adultas junto a Estados Unidos, Canadá, Argentina y Uruguay, con valores entre 6,1 y $8,1 \%$. En Brasil, Perú, Venezuela, Colombia y Cuba la prevalencia de diabetes fue estimada entre 5,1 y 6,0\%, mientras que en Bolivia, Paraguay, Ecuador, Panamá, Costa Rica y Guatemala fue entre 4,1 y 5,0\% y en Surinam, Guyana, Nicaragua y Honduras de entre 3,1 y 4,0\%. Además, se estimó que las poblaciones urbanas (como son aquellas donde se han realizado la mayoría de las encuestas de prevalencia) tenían tasas de prevalencia dos veces mayor que las poblaciones que viven en áreas rurales 8,10 .
Los estudios realizados en Chile en poblaciones aborígenes, y que han sido incluidos en esta revisión de la OMS, han mostrado cambios evidentes en el perfil de obesidad y diabetes tipo 2 en los últimos años. El estudio de Larenas y cols, en 1985, en una comunidad rural Mapuche del sur de Chile, mostró una prevalencia de diabetes de $0,4 \%$ en hombres y $1,4 \%$ en mujeres ${ }^{11}$, sin embargo, la repetición de esta encuesta en 1999, arrojó una prevalencia de diabetes de $3,2 \%$ en hombres y $4,5 \%$ en mujeres ${ }^{12}$, lo que sugiere que está ocurriendo un proceso de aculturización en esta comunidad rural, con un incremento en la prevalencia de diabetes y probablemente de otras enfermedades crónicas. Considerando el mayor riesgo de ECN en zonas urbanas, se espera que estas patologías afecten en mayor proporción a estos grupos cuando viven en ciudades grandes.

El objetivo de esta investigación fue estimar la frecuencia de diabetes tipo 2, obesidad y alteraciones del perfil lipídico en dos poblaciones aborígenes de Chile (comunidades Mapuches y Aymaras) que desarrollan su actividad en un ambiente urbano.

\section{Sujetos y Metodología}

La población objetivo la constituyeron individuos de ambas etnias (Mapuche y Aymara) residentes en sectores urbanos. En el caso de la etnia Mapuche, la investigación se realizó en 4 comunas de la Región Metropolitana (Peñalolén, La Pintana, Cerro Navia y Pudahuel) y la población Aymara urbana que participó de este estudio correspondió a aquella residente en la ciudad de Arica. En ambos casos se tomó contacto con las asociaciones respectivas mediante una charla informativa en la cual se les indicó el objetivo central de pesquisar diabetes tipo 2 .

En ambas localidades (tanto en Santiago como en Arica) no existió un plan de muestreo formal de participantes, sino que éstos fueron incluidos mediante una invitación abierta realizada a cada comunidad indígena en individuos mayores de 20 años. Aunque este procedimiento supone inevitablemente un sesgo de autoselección, pensamos que este factor no ha ejercido una influencia absolutamente decisiva, dado que gran parte de la comunidad participa en estos exámenes periódi- 
cos, independientemente del estado de salud de las personas. En cualquier caso, las conclusiones de esta investigación deben tomarse con precaución y teniendo en cuenta el sistema de reclutamiento empleado. La representatividad de la muestra, desde el punto de vista étnico, se analizó sobre la base de existencia de cuatro apellidos autóctonos y clasificación según grupo sanguíneo ${ }^{13}$. Todos los participantes asistieron en forma voluntaria al consultorio comunal, donde se realizó una prueba de tolerancia oral a la glucosa en condiciones de ayuno y una posterior toma de muestra a los 120 min. Los individuos participantes entregaron su consentimiento escrito respectivo y esta investigación fue aprobada por el Comité de Etica del Servicio de Salud Occidente y formó parte de la ejecución del Proyecto DID en Etnias Indígenas (DID № 022).

Los grupos quedaron constituidos de la siguiente forma: población Aymara $(n=160) 42$ hombres (edad promedio: 52,1 $\pm 14,1$ años, rango: 22-80) y 118 mujeres (edad promedio: 47,5 $\pm 13,6$ años, rango: $21-77)$ y población Mapuche $(\mathrm{n}=147)$ 42 hombres (edad promedio: 45,6 $\pm 13,9$ años, rango: 21-82) y 105 mujeres (edad promedio: $44,3 \pm 14,2$ años, rango: $18-80$ ). La edad promedio fue mayor en el grupo Aymara que en el grupo Mapuche. En forma similar a nuestros estudios previos sobre enfermedades crónicas y pesquisa de diabetes tipo 2 en etnias rurales, la participación de mujeres fue muy superior a la de hombres: $73,8 \%$ en el grupo Aymara y $71,5 \%$ en el grupo Mapuche. Esta mayor participación de mujeres (generada secundariamente al sesgo de autoselección), se debe principalmente a que los hombres no acuden al lugar donde se efectúa el estudio porque en la mayoría de los casos se encuentran en su lugar de trabajo.

Las mediciones antropométricas, peso, talla y circunferencia cintura-cadera (ICC), se realizaron con los individuos descalzos y vestidos con ropa liviana. El índice de masa corporal (IMC) fue utilizado como estimación de la adiposidad calculándose como el peso (kilogramos) dividido por la talla (metros) al cuadrado. Para la clasificación de obesidad se utilizaron criterios de la OMS ${ }^{14,15}$.

La presión arterial sistólica (PS) y diastólica (PD) se determinó con el individuo sentado y en reposo y se consideró el promedio de dos mediciones, una realizada al ingreso y otra una vez finalizada la prueba de tolerancia. Para la clasificación de hipertensión arterial (HTA: PS $\geq 140 \mathrm{mmHg}$ y/o PD $\geq 90 \mathrm{mmHg}$ ) se utilizaron las cifras recomendadas por los consensos internacionales ${ }^{16,17}$.

Todas las determinaciones bioquímicas se realizaron en la muestra tomada en ayunas. La insulina y la leptina plasmáticas se determinaron en duplicado mediante un radioinmunoanálisis específico (DPC Diagnostic y Linco Research), con un coeficiente de variación intraensayo de $2,8 \%$ y un coeficiente de variación interensayo de 3,5\%. Las determinaciones de perfil lipídico, tales como: colesterol total (CT), colesterol HDL (C-HDL) y triglicéridos (TG) se realizaron mediante técnicas colorimétricas estándares. La glicemia se analizó mediante prueba de glucosa-oxidasa. Los criterios utilizados para establecer los respectivos niveles de riesgo según el perfíl lipídico han sido consignados de acuerdo al National Institute of Health de USA y al European Atherosclerosis Group Consensos $^{16,17}$. Finalmente, se utilizó el modelo homeostático HOMA para evaluar insulino-resistencia ${ }^{18}$. Las diferencias estadísticas han sido analizadas mediante la prueba de $t$ de Student 0 Chi cuadrado con el programa Stata 7.0.

\section{RESULTADOS}

La Tabla 1 resume la información ampliada en ambos grupos, observándose que el IMC promedio es mayor entre las mujeres que en los hombres en cada grupo étnico $(p<0,04)$. En términos generales, las mayores diferencias según género se observaron en la población Aymara, donde el grupo de hombres presentó un mayor promedio de edad ( $p<0,01)$, mayor PS ( $p<0,01$ ), mayor PD ( $p<0,035)$. En el grupo de población Mapuche sólo los TG estuvieron aumentados al comparar hombres con mujeres ( $p<0,04)$.

La Tabla 2 muestra la prevalencia de obesidad, HTA, intolerancia a la glucosa y diabetes tipo 2, según sexo en cada grupo étnico. Respecto a la obesidad, empleando el criterio OMS $\left(\geq 30 \mathrm{~kg} / \mathrm{m}^{2}\right)$, se observó claramente una cifra promedio comparable entre ambos grupos étnicos (45\% de obesidad en Aymaras y 48,2\% en Mapuches). En forma similar a nuestros estudios previos en ambiente rural, la obesidad es una característica más fre- 
Tabla 1. Características antropométricas y metabólicas en cada grupo étnico (promedio \pm desviación estándar)

\begin{tabular}{|lcccc|}
\hline & \multicolumn{2}{c}{ Aymara $(\mathrm{n}=160)$} & \multicolumn{2}{c|}{ Mapuche $(\mathrm{n}=147)$} \\
& $\begin{array}{c}\text { Hombres } \\
(\mathrm{n}=42)\end{array}$ & $\begin{array}{c}\text { Mujeres } \\
(\mathrm{n}=118)\end{array}$ & $\begin{array}{c}\text { Hombres } \\
(\mathrm{n}=42)\end{array}$ & $\begin{array}{c}\text { Mujeres } \\
(\mathrm{n}=105)\end{array}$ \\
\hline IMC $\left(\mathrm{kg} / \mathrm{m}^{2}\right)$ & $28,2 \pm 4,1^{*}$ & $30,1 \pm 5,4$ & $29,8 \pm 4,1^{*}$ & $31,8 \pm 5,6$ \\
ICC & $0,94 \pm 0,06$ & $0,87 \pm 0,05$ & $0,92 \pm 0,07$ & $0,87 \pm 0,05$ \\
PS $(\mathrm{mmHg})$ & $121,6 \pm 17,6^{* *}$ & $114,1 \pm 15,5$ & $129,5 \pm 19,8$ & $123,6 \pm 22,6$ \\
PD $(\mathrm{mmHg})$ & $75,1 \pm 9,1^{*}$ & $71,4+9,9$ & $76,3 \pm 11,9 *$ & $72,5 \pm 12,6$ \\
CT $(\mathrm{mg} / \mathrm{dl})$ & $204,1 \pm 36,8$ & $194,5 \pm 40,7$ & $183,5 \pm 45,3$ & $179,6 \pm 48,9$ \\
C-HDL $(\mathrm{mg} / \mathrm{dl})$ & $38,6 \pm 7,8$ & $40,5 \pm 7,8$ & $50,9 \pm 15,9$ & $49,9 \pm 14,4$ \\
TG $(\mathrm{mg} / \mathrm{dl})$ & $138,5 \pm 81,3$ & $122,1 \pm 50,1$ & $148,1 \pm 95,7 *$ & $117,3 \pm 72,2$ \\
Ins $(\mu \mathrm{UI} / \mathrm{ml})$ & $11,8 \pm 9,6$ & $18,7 \pm 24,3$ & $12,8 \pm 16,1$ & $15,7 \pm 22,9$ \\
HOMA IR & $2,4 \pm 1,8$ & $2,8 \pm 5,1$ & $3,5 \pm 6,9$ & $3,3 \pm 5,7$ \\
Leptina $(\mathrm{ng} / \mathrm{ml})$ & $9,8 \pm 6,7$ & $14,5 \pm 9,1$ & $7,4 \pm 5,7$ & $17,6 \pm 8,8$ \\
\hline
\end{tabular}

$*_{p}<0,04$ (comparación hombres versus mujeres en cada grupo).

**p $<0,01$ (hombres versus mujeres, población Aymara).

Tabla 2. Prevalencia de obesidad, hipertensión arterial (H TA), intolerancia a la glucosa (IG) y diabetes tipo 2 en población Aymara urbana $(n=160)$ y en población M apuche urbana $(n=147)$; valores expresados como porcentaje e intervalo de confianza (IC 95\%)

\begin{tabular}{|c|c|c|c|c|}
\hline & \multicolumn{2}{|c|}{ Población Aymara } & \multicolumn{2}{|c|}{ Población Mapuche } \\
\hline & $\begin{array}{l}\text { Hombres } \\
\quad(\mathrm{n}=42)\end{array}$ & $\begin{array}{l}\text { Mujeres } \\
(\mathrm{n}=118)\end{array}$ & $\begin{array}{l}\text { Hombres } \\
(\mathrm{n}=42)\end{array}$ & $\begin{array}{l}\text { Mujeres } \\
(n=105)\end{array}$ \\
\hline & $\mathrm{n} \quad \%$ & $\mathrm{n} \quad \%$ & $\mathrm{n} \quad \%$ & $\mathrm{n} \quad \%$ \\
\hline$\%$ obesidad & $\begin{array}{rr}15 & 35,7 \\
(21,6-51,9)\end{array}$ & $\begin{array}{rr}57 & 48,3 \\
(39,0-57,7)\end{array}$ & $\begin{array}{cr}13 & 30,9 \\
(17,6-47,1)\end{array}$ & $\begin{array}{cr}58 & 55,2^{*} \\
(45,2-64,9)\end{array}$ \\
\hline$\%$ HTA & $\begin{array}{cr}8 & 19,1 \\
(8,6-34,1)\end{array}$ & $\begin{array}{l}5 \quad 4,2 \text { * } \\
(1,3-9,6)\end{array}$ & $\begin{array}{cr}16 & 38,1 \\
(23,5-54,4)\end{array}$ & $\begin{array}{cr}25 & 23,8 \\
(16,0-33,1)\end{array}$ \\
\hline$\%$ IG & $\begin{array}{cr}2 & 4,8 \\
(0,58-16,1)\end{array}$ & $\begin{array}{l}5 \quad 4,2 \\
(1,4-9,6)\end{array}$ & $\begin{array}{lr}3 & 7,1 \\
(1,5-19,5)\end{array}$ & $\begin{array}{l}4 \quad 3,8 \\
(1,1-9,5)\end{array}$ \\
\hline$\%$ diabetes & $\begin{array}{lr}1 & 2,4 \\
(0,1-12,6)\end{array}$ & $\begin{array}{l}10 \quad 8,5 \\
(4,1-15,0)\end{array}$ & 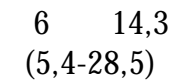 & $\begin{array}{cr}5 & 5,7 \\
(2,1-12,0)\end{array}$ \\
\hline
\end{tabular}

Obesidad $=$ IMC $\geq 30 \mathrm{~kg} / \mathrm{m}^{2}$. HTA $=$ PS $\geq 140 \mathrm{mmHg}$; PS $\geq 90 \mathrm{mmHg} .{ }^{*} \mathrm{p}<0,013$ (obesidad en etnia Mapuche). **p $<0,007$ (HTA en etnia Aymara).

cuente entre las mujeres. En este sentido, se observó un mayor impacto de la obesidad en las mujeres Mapuches (55,2\%), cifra estadísticamente significativa respecto de hombres Mapuches ( $p$ $<0,013)$.

En relación a la prevalencia de HTA, ésta fue significativamente mayor $(p<0,03)$ entre los individuos Mapuches $(27,9 \%)$ que en Aymaras (8,1\%).
La comparación entre hombres y mujeres en cada etnia, mostró una importante diferencia en la prevalencia de HTA en el grupo Aymara (19,1\% en hombres versus $4,2 \%$ en mujeres, $p=0,007)$. El porcentaje de intolerantes a la glucosa fue similar en ambos grupos étnicos (4,3\% en Aymaras y 4,8\% en Mapuches), siendo mayor la frecuencia de intolerantes entre los hombres. 
La prevalencia de diabetes tipo 2 mostró un importante incremento respecto a las cifras publicadas por otros estudios en ambiente rural. En el grupo Aymara, la prevalencia total fue de 6,9\% y en el grupo Mapuche se estimó una prevalencia del $8,2 \%$. El valor de prevalencia estimado para esta investigación ha incluido a todos los diabéticos (conocidos y diagnosticados) involucrados en ella. En el grupo Aymara, del total de 11 diabéticos, 4 de ellos conocían previamente su enfermedad y 7 individuos fueron diagnosticados en terreno. En el grupo Mapuche del total de 12 diabéticos, 6 de ellos estaban en tratamiento y se diagnosticaron 6 nuevos casos.

La Tabla 3 muestra el porcentaje de lípidos alterados en ambos grupos étnicos urbanos. La cifra de colesterol total $\geq 200 \mathrm{mg} / \mathrm{dl}$ fue una característica más frecuente en la población Aymara que en el grupo Mapuche (43,1\% versus $27,9 \%$, $\mathrm{p}<0,008$ ). El colesterol alterado fue un rasgo más prevalente en los hombres Aymaras y en las mujeres Mapuches. El porcentaje de TG alterados ( $\geq 150 \mathrm{mg} / \mathrm{dl}$ ) fue una característica más acentuada en la población Mapuche que en la Aymara $(23,1 \%$ versus $16,9 \%, p=N S)$. En cuanto a los bajos niveles de C-HDL ( $\leq 35 \mathrm{mg} / \mathrm{dl})$, el grupo Aymara presentó una mayor prevalencia respecto al grupo Mapuche $(27,5 \%$ versus $12,9 \%, p<0,002)$. En términos generales, la mayor frecuencia de alteraciones lipídicas se concentró en el grupo de hombres Aymaras.

\section{DisCUSIÓN}

La asociación positiva entre obesidad y riesgo de diabetes tipo 2 es un hallazgo constante en todos los estudios epidemiológicos. Tomando en cuenta que la diabetes está fuertemente asociada con la obesidad y los cambios de peso a través del tiempo, el aumento en las tendencias de obesidad que empiezan en la niñez y adolescencia implica que la diabetes empezará a afectar cada vez a grupos más jóvenes, afectando a las personas durante su período de vida económicamente activo (20-64 años). Además, la diabetes es un importante factor de riesgo para la enfermedad cardíaca, la cual es causa de más de un tercio de todas las muertes observadas en diabéticos mayores de 40 años.

Los graves efectos descritos, hacen extremadamente preocupante el gran aumento de la prevalencia de diabetes tipo 2 que se observa en el mundo entero. Estimaciones globales de la OMS en 1998 calculaban en 143 millones las personas diabéticas en el mundo y 300 millones para el 2025.

Las proyecciones de la OMS respecto de la estructura de edad de la población diabética predice que para el 2025, si persisten las tendencias actuales, la mayoría de las personas con diabetes en las ciudades desarrolladas tendrán 65 o más años, en cambio, la mayoría de los diabéticos en las ciudades en desarrollo pertene-

Tabla 3. Porcentaje de lípidos alterados en ambas poblaciones estudiadas (porcentaje e intervalo de confianza, IC 95\%)

\begin{tabular}{|c|c|c|c|c|}
\hline & \multicolumn{2}{|c|}{ Población Avmara } & \multicolumn{2}{|c|}{ Población Mapuche } \\
\hline & $\begin{array}{c}\text { Hombres } \\
(n=42)\end{array}$ & $\begin{array}{l}\text { Mujeres } \\
(\mathrm{n}=118)\end{array}$ & $\begin{array}{c}\text { Hombres } \\
(n=42)\end{array}$ & $\begin{array}{l}\text { Mujeres } \\
(\mathrm{n}=105)\end{array}$ \\
\hline & $\mathrm{n} \quad \%$ & $\mathrm{n} \quad \%$ & $\mathrm{n} \quad \%$ & n $\%$ \\
\hline CT $\geq 200$ & $21 \quad 50,0$ & $48 \quad 40,7$ & $9 \quad 23,8$ & $31 \quad 29,5$ \\
\hline $\mathrm{mg} / \mathrm{dl}$ & $(34,1-65,8)$ & $(31,7-50,1)$ & $(12,6-39,5)$ & $(21,0-39,2)$ \\
\hline $\mathrm{TG} \geq 150$ & $15 \quad 35,7$ & $12 \quad 10,2 *$ & 1535,7 ** & $19 \quad 18,1$ \\
\hline $\mathrm{mg} / \mathrm{dl}$ & $(21,5-51,9)$ & $(5,3-17,1)$ & $(21,5-51,9)$ & $(11,2-26,8)$ \\
\hline C-HDL $\leq 35$ & $17 \quad 40,4$ & $27 \quad 22,9^{\text {** }}$ & $8 \quad 19,1$ & $10 \quad 10,5$ \\
\hline $\mathrm{mg} / \mathrm{dl}$ & $(25,6-56,7)$ & $(15,7-31,5)$ & $(8,6-34,1)$ & $(5,4-17,9)$ \\
\hline
\end{tabular}

${ }^{*} p<0,001$ (TG hombres versus mujeres, Aymaras). **p $<0,05$ (TG hombres versus mujeres, Mapuches). $* * \mathrm{p}<0,05$ (C-HDL hombres versus mujeres, Aymaras). 
cerán al grupo de 45 a 64 años, en sus años de vida más productivos. De hecho, el mayor aumento en los años de vida saludable (AVISA) perdidos en Latinoamérica al año 2000, está dado por la diabetes ${ }^{19}$.

Estudios conducidos en grupos indígenas chilenos por diferentes investigadores, por lo general han enfocado el problema de las enfermedades crónicas en el ámbito rural. En el año 1985, Larenas y cols ${ }^{11}$, publica los primeros datos de prevalencia de diabetes y obesidad en la etnia Mapuche en una comunidad rural de Ercilla en la IX región. Dicho estudio, determinó en población mayor de 20 años, un porcentaje de obesidad del $17,2 \%$ en hombres y del $61,9 \%$ en mujeres. Un estudio más reciente publicado por Stockins y $\operatorname{cols}^{20}$, publica sobre un excelente tamaño muestral $(\mathrm{n}=1948)$; datos correspondientes al período 1989-1991, observando en población mapuche rural mayor de 25 años un porcentaje de $24 \%$ de obesidad entre los hombres y de $45 \%$ en las mujeres.

Durante el año 1999, nuestro grupo determinó un porcentaje de obesidad en mujeres de $62,9 \%$ y $37,9 \%$ en hombres, también en localidades rurales de la IX región ${ }^{21}$. Los datos presentados en esta investigación, muestran por primera vez una estimación de obesidad en poblaciones autóctonas que habitan un ambiente urbano (población Mapuche de Santiago y población Aymara de Arica)

Las cifras de obesidad publicadas en el año 1990 por Berríos y cols ${ }^{22}$, en población urbana de Santiago de nivel socioeconómico bajo fueron de $13,2 \%$ en hombres y $29,3 \%$ en mujeres. Este estudio reveló una prevalencia de obesidad en el grupo Mapuche de Santiago de 55,2\% en mujeres y $30,9 \%$ en hombres. La población Mapuche de Santiago, se localiza principalmente en comunas populares y de bajo ingreso socioeconómico. Es posible que nuestros resultados estén reflejando el impactante incremento que está teniendo la obesidad en este segmento de la población, aun teniendo en cuenta el posible sesgo de autoselección.

Nuestro estudio ha permitido estimar el porcentaje de obesidad en grupos Aymaras urbanos (localizados en la ciudad de Arica). En forma similar a lo observado en el grupo Mapuche urbano, hemos observado una mayor prevalencia de obesidad en población Aymara urbana, cuando comparamos estos datos con nuestro estudio anterior. Durante 1999, nuestro grupo determinó una prevalencia de $27,0 \%$ en hombres y $39,7 \%$ en mujeres en población Aymara altiplánica ${ }^{21}$. Los resultados obtenidos en esta muestra indican un considerable aumento de la obesidad con cifras de $35,7 \%$ en hombres y $48,3 \%$ en mujeres.

En relación a la HTA, nuestros datos en población Mapuche urbana muestran cifras mayores a las publicadas en años anteriores, detectándose una prevalencia HTA de 38,1\% en hombres y $23,8 \%$ en mujeres, valores superiores a los descritos por nuestro grupo en ambiente rural durante 1999 (20,4\% en hombres y 18,8\% en mujeres) у а los datos publicados por Stockins y cols ${ }^{20}$, donde se observaron cifras de $13,2 \%$ y $16,2 \%$, en hombres y mujeres respectivamente.

Sin embargo, hay que ser cuidadoso en este análisis de HTA, puesto que tanto la prevalencia de nuestro estudio previo ${ }^{21}$ y el trabajo de Stockins y cols ${ }^{20}$, emplearon un punto de corte diferente para la tabulación de HTA. De igual forma, nuestro registro de porcentaje de HTA es mayor que el determinado por Berríos y cols ${ }^{22}$ en población de Santiago (7,7\% en hombres y 11,0\% en mujeres) y mayor al observado por el estudio CARMEN que registró un valor de $13,1 \%$ en hombres y $15,1 \%$ en mujeres en el nivel socioeconómico bajo 23 .

En relación a la HTA, la prevalencia en población Aymara urbana de la ciudad de Arica se encuentra claramente elevada $(19,1 \%$ en hombres y $4,2 \%$ en mujeres) al compararlos con datos publicados en el año 1999 en grupos rurales que indicaron una prevalencia de $9,0 \%$ en hombres y $4,8 \%$ en mujeres. En ambos estudios las cifras son mayores a las reportadas por Cruz-Coke y cols en $1973^{24}$, quienes no encontraron hipertensión en el altiplano $\mathrm{y}$ determinaron una prevalencia del 3,8\% en población Aymara de Azapa. A pesar de que en la actualidad se están utilizando puntos de corte más restrictivos para clasificar HTA, esta tendencia no hace más que confirmar una pionera hipótesis de Cruz-Coke y cols, que indicaba que la HTA se incrementaba cuando la población Aymara emigraba de regiones rurales a zonas más urbanizadas ${ }^{25,26}$.

Otro aspecto importante de esta investigación, fue la determinación de prevalencia de diabetes tipo 2. Si bien, los únicos datos disponibles en la 
literatura en etnias indígenas proceden de ambiente rural, resultaba de gran interés observar el impacto de la obesidad y del ambiente urbano en esta patología. En 1985, Larenas y cols ${ }^{11}$, determinaron una prevalencia de diabetes tipo $2<1 \%$ en población Mapuche rural $(0,4 \%$ en hombres y 0,98\% en mujeres). Durante el año 2001, nuestro grupo publicó el incremento en la prevalencia de diabetes tipo 2 para esta misma etnia en ambiente rural, determinándose una tasa global de 3,85\% $(3,2 \% \text { en hombres y } 4,5 \% \text { en mujeres })^{12}$. Nuestros datos actuales, muestran un incremento notable de la prevalencia en Mapuches de la ciudad de Santiago, alcanzando un valor de $8,2 \%(5,7 \%$ en mujeres y $14,3 \%$ en hombres) ( $p<0,02$ ). Claramente el grupo más afectado en esta etnia son los hombres, quienes además muestran el mayor incremento de obesidad.

En la población Aymara, se observó una situación similar. Nuestro estudio, publicado en 2001, en población rural, mostró una prevalencia global de diabetes tipo 2 de 1,5\% (1,3\% en hombres y $1,7 \%$ en mujeres) ${ }^{9}$. La prevalencia total determinada en esta muestra urbana fue significativamente mayor $6,9 \%$ ( $p<0,01)(2,4 \%$ en hombres y $8,5 \%$ en mujeres), cifra que se aproxima más a los valores publicados para población mixta de Santiago y Valparaíso ${ }^{23,27}$.

En este mismo contexto, durante el año 2002 Baechler y cols ${ }^{28}$ informan sobre la prevalencia de diabetes tipo 2 en la VII región de Chile, detectando prevalencias del 5,8\% en el ámbito urbano y del 4,5\% en el ambiente rural, una tendencia muy similar a la observada en nuestro estudio, que da cuenta del aumento en la frecuencia de diabetes tipo 2 en ambiente urbano.

Considerando algunos indicadores de riesgo asociados a metabolismo de lípidos, en el grupo Mapuche urbano, el colesterol por sobre los 200 $\mathrm{mg} / \mathrm{dl}$ se presentó en $27,9 \%$ de los individuos, cifra muy similar a la descrita por Stockins y cols $(31,2 \%)^{20}$ y por los estudios de Berríos y cols $(36,9 \%)^{22}$. La población Aymara presentó un patrón lipídico alterado para colesterol total muy similar entre ambos sexos (promedio 43,1\%). En relación a los otros parámetros lipídicos los TG alterados mostraron una distribución similar tanto en Mapuches como en Aymaras urbanos. Lamentablemente, los estudios anteriores utilizaron como punto de corte el valor de $250 \mathrm{mg} / \mathrm{dl} \mathrm{y}$ nuestras cifras de TG alterados no pueden ser comparadas.

Finalmente, el porcentaje de colesterol HDL $\leq 35 \mathrm{mg} / \mathrm{dl}$ fue más prevalente en el grupo Aymara $(27,5 \%)$ que en el grupo Mapuche (12,9\%). Datos de nuestro estudio anterior mostraron que 14\% de la población Mapuche rural presentaba esta alteración ${ }^{21}$. Estudios publicados con anterioridad 20,22 en Mapuches y en Santiago, informaron de un porcentaje de colesterol HDL inferior a los $35 \mathrm{mg} /$ dl en el $12,6 \%$ y $19,6 \%$ de los hombres y en el $9,6 \%$ y $10,2 \%$ de las mujeres respectivamente. Para la población Aymara rural, los porcentajes de colesterol HDL bajo los $35 \mathrm{mg} / \mathrm{dl}$ fueron comparables a los determinados por este estudio en el ámbito urbano (25,6\%). En términos comparativos, la población Aymara de Arica presentó un mayor perfil de riesgo lipídico asociado a bajos niveles de colesterol HDL y elevado colesterol total en comparación al grupo Mapuche urbano.

Existe claridad respecto a que el grupo de estudio involucrado en esta investigación es inferior en tamaño muestral a los anteriormente publicados, no obstante, el diseño de pesquisa y la metodología empleada es similar. No cabe duda que la ampliación de la muestra permitiría obtener conclusiones más definitivas, sin embargo existe una dificultad mayor para trabajar con etnias urbanas (Santiago y Arica) principalmente debido a que en las grandes ciudades estas comunidades se encuentran más disgregadas y con un mayor componente de mezcla.

La tendencia observada en la variación de la prevalencia de obesidad, HTA y fundamentalmente diabetes tipo 2, muestran claramente que en estas comunidades, cuya prevalencia de obesidad es comparable a la observada en etnias rurales 9,12 , se observa un mayor impacto en el diagnóstico de diabetes tipo 2, encontrándose cifras de prevalencia que incluso superan a las informadas en población caucásica de Santiago y Valparaíso 23,27. Al respecto, varios factores podrían tener relación con el aumento en las cifras de diabetes en poblaciones migrantes, una de ellas podría ser la actividad física y el probable mayor grado de sedentarismo que estaría afectando a las comunidades autóctonas urbanas. Otro elemento relevante lo constituye la dieta. Si bien no hay un análisis exhaustivo respecto a posibles diferencias entre dieta «ural»y dieta «urbana», podría ser un 
componente gravitante desde el punto de vista del aporte de grasas saturadas y nutrientes específicos que modifican el metabolismo de la glucosa. Finalmente, no hay que descartar la posibilidad del impacto que podrían tener los denominados genes ahorradores (thrifty genes) que indica que individuos expuestos a períodos de restricción alimentaria, pueden mantener por generaciones cierta «resistencia» al desarrollo de patologías crónicas, por un mecanismo adaptativo que impi-

\section{REFERENCIAS}

1. Ogden CL, Carroll MD, Fiegal KM. Epidemiologic trends in overweight and obesity. Endocrinol Metab Clin N Am 2003; 32: 741-60.

2. Lewis G, Carpentier A, Adeu K, Giacca A. Disordered fat storage and mobilization in the pathogenesis of insulin resistance and type 2 diabetes. Endocrine Reviews 2002; 23: 201-29.

3. BerRíos X. Tendencia temporal de los factores de riesgo de enfermedades crónicas: da antesala silenciosa de una epidemia que viene? Rev Méd Chile 1997; 125: 1405-7.

4. Rozowski J, Arteaga A. El problema de la obesidad y sus características alarmantes en Chile. Rev Méd Chile 1997; 125: 1217-24.

5. Aibala C, Vio F, Kain J. Obesidad: un desafío pendiente en Chile. Rev Méd Chile 1998; 126: 1001-9.

6. Albala C, Vio F, Kain J, Uauy R. Nutrition transition in Chile: determinants and consequences. Public Health Nutr 2002; 5: 123-8.

7. Albala C, Vio F, Kain J, Uauy R. Nutrition transition in Latin America: the case of Chile. Nutr Rev 2001; 59: 170-6.

8. BARCeló A. La diabetes en las Américas. Boletín Epidemiológico OPS 2001; 22: 1-3.

9. Santos Jl, Pérez-Bravo F, Carrasco E, Calvilán M, Aibaia C. Low prevalence of type 2 diabetes despite a high average body mass index in the Aymara natives from Chile. Nutrition 2001; 17: 305-9.

10. King H, Aubert RE, Herman WH. Global Burden of Diabetes, 1995-2025. Diabetes Care 1998; 21: 1414-31.

11. Larenas G, Arias G, Espinoza O, Charies M, Landaeta O, Villanueva $S$ et al. Prevalencia de diabetes mellitus en una comunidad Mapuche de la IX Región, Chile. Rev Méd Chile 1985; 113: 1121-5. de expresar ciertas familias de genes. No obstante, bajo situaciones de exceso (grasas saturadas, productos refinados) este genotipo protector no responde y se manifiesta con un potencial agravante mayor ${ }^{29-31}$. Si bien este último factor es más especulativo, permitiría entender el por qué poblaciones genéticamente similares, con un porcentaje comparable de obesidad y que habitan ambientes diferentes, presentan un patrón tan distinto en la frecuencia de diabetes tipo 2.

12. Pérez-Bravo F, Carrasco E, Santos JL, Calvilán M, Larenas G, Albala C. Prevalence of type 2 diabetes and obesity in rural Mapuche population from Chile. Nutrition 2001; 17: 236-8.

13. ValenZueia C, AcuÑa M, HaRB Z. Gradiente sociogenético en la población chilena. Rev Méd Chile 1987; 115: 295-9.

14. WHO Consultation 1999. Definition, diagnosis and classification of diabetes mellitus and its complications. WHO/NCD/NCS/99.2:31-3.

15. Wordd Health Organization (WHO). Obesity: preventing and managing the global epidemic. Report of a WHO consultation on obesity. WHO/ NUT/NCD/98.1.1997.

16. World Health Report 2002. Reducing Risks. Promoting Healthy Life. Geneva Switzerland: World Health Organization; 2002; 58.

17. 2003 European Society of Hypertension-European Society of Cardiology guidelines for the management of arterial hypertension Guidelines Committee. J Hypertension 2003; 21: 1011-53.

18. Matthews DR, Hosker JP, Rudenski AS, Naylor BA, TREACHER DF, TurNer RC. Homeostasis model assessment: insulin resistance and beta-cell function from fasting plasma glucose and insulin concentrations in man. Diabetologia 1985; 28: 412-9.

19. Albala C, Vio F, Uauy R. The global burden of nutritional disease: The Case of Latin America. En: The control of Food and Fluid Intake in Health and Disease. Nestlé Nutrition Workshop Series Pediatric Program. Farthing M, Mahalanabis D (Eds). 2003; 87-102.

20. Stockins B, Larenas G, Charles M, Standen D, Espinoza O, ILesca M et al. Niveles de lípidos y de presión arterial en población Mapuche de la región de la Araucanía, en Chile. Rev Méd Chile 1998; 126: 1291-9. 
21. Pérez F, Carrasco E, Santos Jl, Calvilúa M, Albala C. Prevalencia de obesidad, hipertensión arterial y dislipidemia en grupos aborígenes rurales de Chile. Rev Méd Chile 1999; 127: 1169-75.

22. Berríos X, Jadue L, Zenteno J, Ross MI, RodrígueZ H. Prevalencia de factores de riesgo de enfermedades crónicas. Estudio en población general de la Región Metropolitana, 1986-1987. Rev Méd Chile 1990; 118: 597-604.

23. Vega J, Jadue L, Escobar MC, Jalll J, Espejo F, Delgado I ET al. Prevalencia de hipertensión arterial en Valparaíso. Resultados de la encuesta de base del programa CARMEN. Rev Méd Chile 1999; 127: 729-38.

24. Cruz-Coke R, Donoso H, Barrera R Genetic ecology of hipertension. Clin Sci Mol Med 1973; 45: 55-65.

25. CRUZ-CoKe R, ETCheverRy R, NAgel R. Influence of migration on blood pressure of Easter islanders. Lancet 1964; I: 697-9.

26. CRuz-Coкe R. Correlation between prevalence of hipertension and degree of acculturation. J Hypertension 1987; 5: 47-50.
27. Melia I, García de los Ríos M, Parker M, CovarruBIAS A. Prevalencia de Diabetes mellitus no insulino-dependiente en el Gran Santiago. Rev Méd Chile 1981; 109: 869-75.

28. Baechler R, Mujica V, Aqueveque X, Ramos L, Soto A. Prevalencia de diabetes mellitus en la VII región de Chile. Rev Méd Chile 2002; 130: 1257-64.

29. Diamond J. The double puzzle of diabetes. Nature 2003; 423: 599-602.

30. Maggio CA, Pi-Sunyer FX. Obesity and type 2 diabetes. Endocrinol Metab Clin N Am 2003; 32: 805-22.

31. Loos RJF, Bouchard C. Obesity: is it a genetic disorder? J Intern Med 2003; 254: 401-25.

Agradecimientos:

Los autores agradecen la colaboración de la Corporación Nacional de Desarrollo Indígena (CONADI), que permitió la coordinación comunal para la realización de los diferentes exámenes. Este proyecto fue financiado a través del proyecto DI 022 (etnias indígenas) de la Universidad de Chile. 